\title{
Neonates and COVID-19: state of the art
}

\section{Neonatal Sepsis series}

\author{
L. Ryan ${ }^{1}$, Frans B. Plötz ${ }^{2,3}$, Agnes van den Hoogen ${ }^{4}$, Jos M. Latour ${ }^{5}$, Marina Degtyareva ${ }^{6}$, Maya Keuning ${ }^{3}$, Claus Klingenberg ${ }^{7,8}$, \\ Irwin K. M. Reiss ${ }^{9}$, Eric Giannoni ${ }^{10}$, Charles Roehr ${ }^{11}$, Christopher Gale ${ }^{12}$ and Eleanor J. Molloy (iD ${ }^{1,13,14,15,16,17 凶}$
}

(c) The Author(s), under exclusive licence to the International Pediatric Research Foundation, Inc 2021

\begin{abstract}
The SARS-CoV-2 pandemic has had a significant impact worldwide, particularly in middle- and low-income countries. While this impact has been well-recognized in certain age groups, the effects, both direct and indirect, on the neonatal population remain largely unknown. There are placental changes associated, though the contributions to maternal and fetal illness have not been fully determined. The rate of premature delivery has increased and SARS-CoV-2 infection is proportionately higher in premature neonates, which appears to be related to premature delivery for maternal reasons rather than an increase in spontaneous preterm labor. There is much room for expansion, including long-term data on outcomes for affected babies. Though uncommon, there has been evidence of adverse events in neonates, including Multisystem Inflammatory Syndrome in Children, associated with COVID-19 (MIS-C). There are recommendations for reduction of viral transmission to neonates, though more research is required to determine the role of passive immunization of the fetus via maternal vaccination. There is now considerable evidence suggesting that the severe visitation restrictions implemented early in the pandemic have negatively impacted the care of the neonate and the experiences of both parents and healthcare professionals alike. Ongoing collaboration is required to determine the full impact, and guidelines for future management.
\end{abstract}

Pediatric Research (2022) 91:432-439; https://doi.org/10.1038/s41390-021-01875-y

IMPACT:

- Comprehensive review of current available evidence related to impact of the COVID-19 pandemic on neonates, effects on their health, impact on their quality of care and indirect influences on their clinical course, including comparisons with other age groups.

- Reference to current evidence for maternal experience of infection and how it impacts the fetus and then neonate.

- Outline of the need for ongoing research, including specific areas in which there are significant gaps in knowledge.

\section{INTRODUCTION}

The SARS-CoV-2 pandemic has impacted the global community with disastrous economic consequences, disrupted social structures, and strained healthcare capacities in both high ${ }^{1}$ and lowand middle-income countries (LMIC). ${ }^{1,2}$ As in high-income countries, COVID-19 in children remains less severe than in the adult population; however, the absolute pediatric fatality rates are disproportionately highest in LMICs. ${ }^{3-5}$ In contrast to older children and adults, COVID-19 in neonates remains uncommon. ${ }^{6}$ However, neonates can be affected by SARS-CoV-2 indirectly, through the impact of maternal COVID-19 during pregnancy, for example leading to preterm birth. Vertical transmission is considered rare, and postnatal infections are equally seen in breastfed and formula-fed infants. ${ }^{7}$ Despite intense research, it remains unclear why neonates mainly experience mild symptoms and have lower mortality rates. 6,8

\section{EPIDEMIOLOGY}

There is a paucity of epidemiological data on neonatal COVID-19. A population-based study of SARS-CoV-2 infection in neonates from the UK, a country that has been severely affected by the

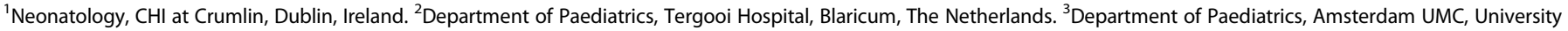

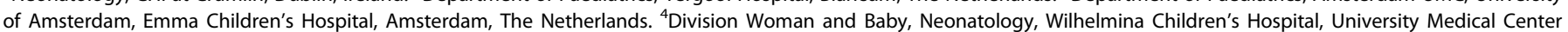

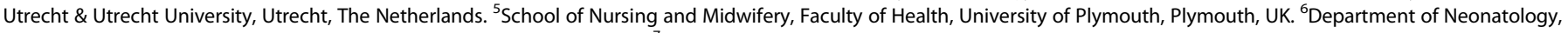

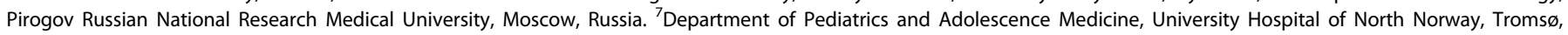

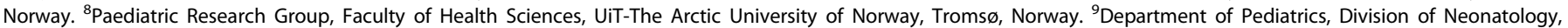

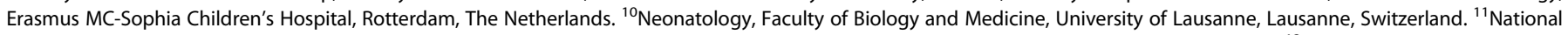

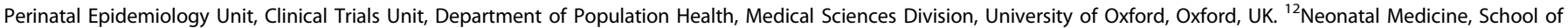

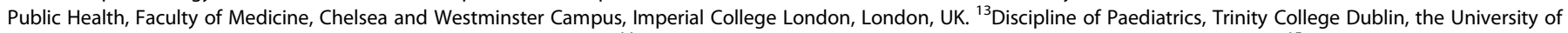

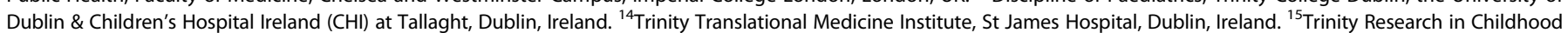

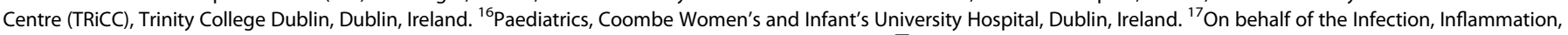
Immunology and Immunization (14) section of the European Society for Paediatric Research (ESPR) ${ }^{\bowtie}$ email: Eleanor.molloy@tcd.ie
} 
pandemic found that during the first wave of the pandemic, 66 babies with confirmed SARS-CoV-2 infection (incidence 5.6 per 10,000 livebirths) received inpatient care. ${ }^{9}$ Population-level in UK demonstrates that SARS-CoV-2 infection is more common in babies from Black (18.0 [7.8-35.5] per 10,000 livebirths) and Asian (15.2 [8.3-25.5] per 10,000 livebirths) ethnic groups when compared to babies from white ethnic groups (4.6 [3.2-6.4] per 10,000 livebirths), in keeping with patterns seen in other age groups. ${ }^{9,10}$ SARS-CoV-2 infection is also more common in babies born preterm; incidence of 18.4 (9.8-31.4) per 10,000 livebirths in babies born between 32 and 37 gestational weeks compared with 4.9 (3.6-6.5) per 10,000 in term babies. ${ }^{9}$ In Norway, a country less severely affected by the pandemic, only three babies with a SARSCoV-2 infection were admitted to a neonatal unit in 2020, all with very mild symptoms [incidence 0.8 per 10,000 livebirthsunpublished data (correspondence CK)]. Globally, there are still knowledge gaps in the epidemiology, clinical manifestations, and outcomes of SARS-CoV-2 infection among neonates.

The clinical presentation in neonates appears different to older children and adults, with gastrointestinal signs and poor feeding more commonly seen. ${ }^{11,12}$ Short-term outcomes of neonatal SARSCoV-2 infection to date are good, with no deaths attributable to SARS-CoV-2 infection noted in UK data. ${ }^{9}$ The longer-term neurodevelopmental impact of neonatal and antenatal exposure to SARS-CoV-2 is currently unknown. In view of the neurotropic potential of the SARS-CoV-2 virus in other age groups, ${ }^{13}$ ongoing neurodevelopmental follow-up of antenatally and neonatally exposed infants is advisable. This should ideally be performed through an international, coordinated, prospective cohort study. ${ }^{14}$

The indirect impact of maternal SARS-CoV-2 infection on the neonate is also poorly characterized but may be considerable. Population-level data from the UK Obstetric Surveillance System identified 640 completed pregnancies, (1.7 per 1000 maternities), in women who had symptomatic SARS-CoV-2 infection in pregnancy during the first 6 months of the UK pandemic (1st March 2020 to 31st August 2020), and 627 live-born infants. A total of $19 \%$ of infants were born preterm (compared to UK preterm birth rates of $7.8 \%), 14 \%$ were iatrogenic preterm births and $19 \%$ of infants received neonatal care. ${ }^{7,9}$ Other indirect effects include, with diversion of resources, shortage of qualified perinatal staff, and fear among pregnant mothers to seek healthcare, which are also of great concern for global neonatal health. ${ }^{15,16}$ A survey among healthcare providers in LMICs showed significant challenges to neonatal care, particularly in the poorest countries. ${ }^{16,17}$ Respondents noted exacerbations of pre-existing shortages in staffing, equipment, and isolation capabilities. In sub-Saharan Africa, a quarter of respondents reported increased mortality in non-COVID-19-infected infants. They also reported decreased admission rates to the neonatal unit during the pandemic, also described in other low-resource settings. ${ }^{15,16}$ In a large observational study from Nepal, institutional childbirth was reduced by more than half during the pandemic lockdown, with increases in institutional stillbirth rate and neonatal mortality, and decreases in quality of care. ${ }^{18}$ Collectively, these are alarming observations of diversion of physical, financial, and personnel resources away from neonates during the pandemic, severely threatening global neonatal health. ${ }^{19-22}$

\section{PLACENTAL AND CONGENITAL OR PERINATAL SARS-COV-2 INFECTION}

Pregnant women are considered to be a high-risk group as they are more likely to require intensive care for COVID-19 compared with non-pregnant women of similar age. ${ }^{23}$ SARS-CoV-2 infection during pregnancy can potentially impact the health of fetuses and neonates through different mechanisms: increased rates of preterm birth, placental infection which may compromise gas and nutrient exchange, leading to intrauterine death or perinatal asphyxia, and through transmission of the virus in utero, during delivery or after birth. Further, the potential effects of maternal medical treatment for SARS-CoV-2 infection, including vaccination, on the fetus remain largely unknown. At this time, there is limited data on the epidemiology of placental and perinatal SARS-CoV-2 infection, due to the lack of data, the limitations of diagnostic tests, and the lack of standardized definitions. To address these issues, the WHO has recently proposed a classification system for congenital, perinatal and postnatal transmission of SARS-CoV-2. ${ }^{24}$

The overall rate of preterm birth is $17 \%$, corresponding to a threefold increase in the rate of preterm birth compared to the general population. ${ }^{23}$ Most preterm births related to COVID-19 are medically induced, due to maternal illness, with no known increase in the rate of spontaneous preterm birth. Reports on the rate of stillbirth related to COVID-19 have shown conflicting results. ${ }^{25-27}$ Some have suggested an increase, but that may be related to disruptions in prenatal care and a higher frequency of home births, or may be coincidental, in light of their small numbers.

Overall, short-term outcomes of infants born to mothers who developed COVID-19 during pregnancy seem favorable. Rates of transmission of SARS-CoV-2 are estimated at 1.9 per 100 pregnancies. Postnatal transmission of SARS-CoV-2 accounts for the majority of infections reported in neonates. Adverse neonatal outcomes of infants of COVID-19 mothers, such as death, have been mainly attributed to prematurity or comorbidities. However, the burden of disease should not be minimized, as $25 \%$ of neonates born to mothers infected with COVID-19 are admitted to a neonatal unit, which corresponds to a threefold increase in the rate of neonatal hospitalization. ${ }^{23}$ Moreover, several cases of severe neonatal disease, including perinatal asphyxia, respiratory failure, multiorgan dysfunction, brain damage and death, have been reported. ${ }^{28-31}$ These cases were mainly related to suspected or proven congenital SARC-CoV-2 infection, with or without infection of the placenta by SARS-CoV-2, or to placental SARS-CoV2 infection without congenital infection.

As SARS-CoV-2 has been detected in the circulation of infected adults, it is hypothesized that the virus can reach the placenta through haematogenous spread. Its presence has been detected in placental tissue by RT-PCR, RNAscope and electron microscopy, ${ }^{28,32-35}$ localized predominantly to syncytiotrophoblasts but also to other placental cell types. ${ }^{36,37}$ Similar to other manifestations of COVID-19, placental infections lead to a spectrum of disease severity, both for the mother and the fetus. ${ }^{36}$ The histopathological features of placental SARS-CoV-2 infection during the second and third trimester are heterogeneous, showing varying degrees of inflammation and vascular malperfusion, mainly on the maternal side. Vascular malperfusion of the placental bed in COVID-19 can result from systemic effects of SARS-COV2 on maternal vasculature and/or invasion of the placenta by the virus. Placental SARS-CoV-2 infection can lead to intense inflammation of the maternal compartment of the placenta, i.e. the intervillous space. This inflammation and necrosis can compromise the function of the placenta, leading to adverse outcomes for the fetus and neonate. A compromised placental barrier could also facilitate the transfer of the virus from the mother to the fetus.

SARS-CoV-2 is commonly found in the stools of infected subjects. ${ }^{38}$ Fecal contamination of the birth canal during labor and delivery could potentially lead to viral infection of the neonate. But transmission of the virus from the mother or other sources in the immediate postpartum period may lead to a similar clinical picture. Placental, congenital and perinatal infections are rare complications of maternal SARS-CoV-2 during pregnancy and in most cases, symptoms are minimal. However, adverse perinatal outcomes such as stillbirth, fetal growth restriction, perinatal asphyxia and severe neonatal pulmonary and systemic disease have been reported. The long-term consequences are unknown. 


\section{POTENTIAL STRATEGIES IN PREVENTION OF PERINATAL AND NEONATAL COVID-19}

Protection of the pregnant, postpartum and breastfeeding female is the first step to preventing placental dysfunctions and disease for the fetus and newborn. ${ }^{39}$

Observational studies confirm that parental triage on arrival at the neonatal ward, universal testing with nasopharyngeal swabs and blood testing for SARS-CoV-2 IgM and IgG antibodies, the continuous use of personal protective equipment at the NICU by parents and staff as well as stringent infection control procedures promote prevention of horizontal, nosocomial transmission of SARS-CoV-2 infection and non-COVID comorbidity among the neonates admitted to a NICU, especially those with pre-morbid conditions. ${ }^{40-42}$ According to observational prospective cohort studies, neonates roomed-in with SARS-CoV-2-positive mother had higher transmission risk; and SARS-CoV-2-positive neonates were more likely to be symptomatic and need resuscitation compared to SARS-CoV-2-negative neonates. ${ }^{43,44}$ Despite the fact that early separation of the father can protect the newborns from possible horizontal transmission of SARS-CoV-2, it is not effective at preventing antenatal and intrapartum vertical transmission. ${ }^{9,45,46}$ It is also associated with increased maternal stress, risk for traumatic childbirth, further maternal psychiatric morbidity ${ }^{47-49}$ and can negatively affect breastfeeding and mother-neonate bonding. ${ }^{45,46}$ Protected rooming-in is an evidence-based preventive measure for neonates born to mothers with SARS-CoV-2 infection. It is based on overall clinical context and is preceded by an educational program, including handwashing, use of surgical face masks during breastfeeding or caring for the infant, and otherwise physical distancing $(2 \mathrm{~m})$ from the infant, strict adherence to sterilization guidelines policies while expressing breastmilk, infection control and prevention practices at home. ${ }^{50,51}$

Maternal neutralizing anti-SARS-CoV-2 adaptive antibodies, including IgG to RBD of the viral spike protein, are known to transfer across the placenta after asymptomatic and symptomatic infection during pregnancy; though no association of antibody concentrations with neonatal outcomes has been provided yet. Transfer ratios increase with increasing time between onset of maternal infection and delivery with maximum at 60-180 days before delivery. ${ }^{52-55}$ Thus, maternal vaccination may protect the infant after an adequate interval from vaccination to delivery (of at least 4 weeks). ${ }^{56}$ Maternally derived anti-SARS-CoV-2 IgG may persist in infants up to 6 months of life. ${ }^{52}$

Transplacental passage of anti-SARS-CoV-2 lgG was at first shown after vaccination in the third trimester of pregnancy. ${ }^{57}$ Maternal immunization may provide neonatal protection through the transplacental transfer of antibodies; antibody transfer ratio being correlated with the time from vaccination to delivery. ${ }^{58}$

Preventive effects of exclusive breastfeeding should be considered in all cases unless contraindications exist. Evidence of SARS-CoV-2 RNA detection in human milk is limited with no information about viral infectivity and no clinical significance for the infant. ${ }^{59-66}$ The preventive role of breastfeeding is based on a robust slgA-dominant SARS-CoV-2 antibody response in human milk after maternal infection with specific reactivity to the full SARS-CoV-2 Spike protein, to its Receptor-Binding Domain (slgA, IgG and/or IgM), S1 or S2 subunit and nucleocapsid from SARSCoV-2. ${ }^{67-74}$ slgA against various SARS-CoV-2 epitopes [N-protein; S-protein linear epitopes (NTD, RBD-SD1) and S-protein conformational RBD epitopes] were found in convalescent donor milk, capable to neutralize viral activity and limit intestinal inflammation. ${ }^{68-70,75-82}$ Lactoferrin prevents viral anchoring on host cell receptors and its concentration in breastmilk is negatively influenced by the severity of maternal COVID-19 infection during pregnancy. ${ }^{83}$

Holder pasteurization $\left(62.5^{\circ} \mathrm{C}\right.$ for $30 \mathrm{~min}$ ) prevents SARS-CoV-2 transmission through human milk, with partial loss of endogenous lysozyme, lactoperoxidase, lactoferrin, and reduction of neutralization capacity of SARS-CoV-2-specific IgA, while high-pressure pasteurization preserves this function. ${ }^{39,71-73,84,85}$

Recent studies show that receipt of a COVID-19 mRNA vaccine was immunogenic in pregnant and lactating women. ${ }^{67,86-89}$ Binding, neutralizing, and functional non-neutralizing antibody responses as well as CD4 and CD8 T-cell responses were present in pregnant, lactating, and non-pregnant women following vaccination, and vaccine-elicited antibodies were transported to infant cord blood and breastmilk. Pregnant and non-pregnant women who were vaccinated developed cross-reactive antibody responses and T-cell responses against SARS-CoV-2 variants of concern. ${ }^{86,87}$ Vaccine-induced immune responses were significantly greater than the response to natural infection. ${ }^{87}$ Spikespecific lgG was thought to dominate after COVID-19 vaccination, unlike the post-infection milk antibody profile with predominant $\operatorname{lgA}{ }^{67,74}$ After vaccination with the mRNA-based BNT162b2 vaccine, a SARS-CoV-2-specific antibody response was detected in human milk. The presence of SARS-CoV-2-specific $\operatorname{lgA}$ after vaccination is important as antibodies are transferred via human milk, and provide protection to infants against COVID-19. SARSCoV-2-specific IgA against the spike protein starts to increase between days 5 and 7 after the first dose of vaccine, an accelerated $\lg \mathrm{A}$ antibody response is observed after the second dose. $^{90}$ The potential protection of breastfed infants by administration of the BNT162b2 COVID-19 vaccine to the breastfeeding mother was demonstrated. ${ }^{88}$ Vaccine-related mRNA is not transferred to the infant, and lactating women who receive the COVID-19 mRNA-based vaccine should not stop breastfeeding during vaccination with COVID-19 mRNA-based vaccine. ${ }^{91} 85 \%$ of breastfeeding women who received an mRNA COVID-19 vaccine reported local or systemic symptoms, with higher frequency following the second dose. Few symptoms were reported in their breastfed children. No serious adverse events were noted. ${ }^{92}$ COVID-19 vaccination of breastfeeding mothers resulted in minimal disruption of lactation or adverse impact on the breastfed child. ${ }^{93}$

Clinical data from larger populations are needed to better estimate the prophylactic effect of the vaccines on short-term and long-term lactation and neonatal clinical outcomes as well as persistence of cellular and humoral SARS-CoV-2-specific immunological memory after infection or vaccination, likely contributing towards protection against reinfection. ${ }^{94}$

\section{PARACOVID EFFECTS AND SEPARATION FROM PARENTS}

In the emergency situation of the COVID-19 pandemic, decisions were made swiftly with little evidence to support them. Limitations in visiting policies and consequently separation of infants and parents in the neonatal intensive care units (NICUs) was observed globally. Hospitals and healthcare services implemented restricted visitation policies in an attempt to control SARS-CoV-2 spread by limiting access to patients, with exception of necessary staff.95,96 These policies affected the care within the concepts of family centered (FCC) and family integrated care (FIC), including breastfeeding, skinto-skin care and zero separation by family closeness. ${ }^{97-99}$ These models of care within neonatology exist to encourage and empower parents to participate in the care of their infants and improve clinical outcomes. ${ }^{100}$ Studies carried out during the COVID-19 pandemic are showing how daily care practices have been influenced by parental presence, developmental care and breastfeeding support. ${ }^{95,100}$ The COVID-19 pandemic and the alterations in FCC and FIC also resulted in parental mental health issues with feelings of fear and sadness increasing the risk for posttraumatic stress and postnatal depression. ${ }^{100-103}$ Van Veenendaal et al. $^{95}$ confirmed that visitation restrictions also had an impact on healthcare professionals, including lack of personal protective equipment, staff shortages and staff concern about the lack of parental presence in the NICU, leading to 
high levels of stress and anxiety amongst staff. The disruption of FCC and FIC, including kangaroo or skin-to-skin care and family closeness, might outweigh the small risk of death due to the virus infection. ${ }^{104}$ Sustainable close contact between these infants and their parents is crucial. $^{104}$ In addition, mothers reported that they stopped breastfeeding because of reduced support or of safety concerns. ${ }^{100}$ All of these observed changes led to the European Foundation for the Care of Newborn Infants (EFCNI) to call for a Zero Separation policy between parents and infants to avoid unnecessary suffering and deaths of the youngest and most vulnerable members of society. ${ }^{105}$ The EFCNI Zero separation campaign increased the awareness of all healthcare professionals in neonatal care to apply FCC and FIC as essential concepts for good neonatal care. This includes supporting early breastfeeding, skin-to-skin care, parental presence and involvement. ${ }^{106-108}$

\section{WHY DON'T NEONATES GET COVID?}

Newborn infants have an altered immunophenotype that changes in early life to resemble that of adults. ${ }^{109,110}$ In children less than 18 years, only $2 \%$ were severely affected by SARS-CoV-2. ${ }^{111}$ However, neonates and children are usually more susceptible than adults to infection and sepsis with increased morbidity and mortality from bacteria, fungi and viruses. Although babies have passive immunity from their mother, this provides little protection from RSV and SARS-CoV-2. Maternally derived immunoglobulin is similar in term and preterm infants and does not explain the differences in susceptibility to infection including viruses like RSV, CMV etc. ${ }^{112}$ In addition, mothers can be severely affected but the baby has minimal signs of illness. ${ }^{113}$ Immune system development continues until the first years of life and is impacted by the microbiome composition of the mother and infant.

Although the relative difference in ACE receptors, which is the main receptor for the entry of SAR-CoV-2 into cells, has been suggested to be a contributory factor in neonatal resistance to COVID-19, there is not sufficient supporting evidence. ACE receptors are considered to be altered in children and there is decreasing ACE2 with age in animal models. ${ }^{114}$ However, results are controversial in humans and recombinant ACE2 is associated with decreased severity of RSV-associated lung injury in an animal model. ${ }^{115}$

The immune response in COVID-19 is predominantly neutrophilic, which is unusual for a viral infection, and the stronger innate immune response with less adaptive immune development may prevent hyperinflammation and a cytokine storm in children. Toll-like receptors activate a number of downstream pathways to initiate an immune response, ${ }^{116-119}$ and are implicated in neonatal disorders including necrotizing enterocolitis, sepsis and periventricular leukomalacia. TLRs induce type I IFNs, which are the body's first defense against viral infections, and TLR4-induced IFN- $\beta$ is decreased in cord blood.

It is suggested that the increased susceptibility of olders adults to COVID 19 may be related to a chronic low-grade systemic inflammation (inflammaging) with higher plasma levels of IL-6, TNF- $a$, and other innate cytokines. ${ }^{120}$ Differences in immune responses in neonates from adults have been attributed to other factors such as relative vitamin D deficiency in adults, increased comorbidities and endothelial damage, chronic altered density and distribution of ACE receptors ${ }^{121-124}$ and Cytomegalovirus infection.

\section{NEONATAL MULTISYSTEM INFLAMMATORY SYNDROME (MISC/PIMS-TS)}

A post-viral syndrome is now recognized in children called multisystem-inflammatory syndrome temporally associated with COVID19, also known as PIMS-TS. ${ }^{125}$ The clinical presentation overlaps with Kawasaki syndrome and Toxic Shock syndrome, and may be considered as part of a spectrum involving these previously known conditions. It usually affects children in later childhood and is characterized by fever, elevated inflammatory biomarkers and organ dysfunction, with particular focus on involvement of the cardiovascular system, including shock, hypotension, myocardial dysfunction. ${ }^{125}$ Only $4 \%$ of cases of MISC were in infants $<1$ year (CDC) ${ }^{45}$ Although rare, there have been case reports of neonatal MISC. ${ }^{126-132}$ Case series and reports have described a variety of clinical features in newborns with COVID19 from thrombosis to atrioventricular conduction defects and have been well-described and illustrated by Sankaran et al. and Pawar et al. ${ }^{126,133}$ The treatment options, largely derived from evidence from trials in adults, ${ }^{134}$ involve dexamethasone and immunoglobulins and require individualized care and careful cardiac evaluation, including close follow-up after clinical improvement and discharge home.

\section{FUTURE DIRECTIONS/PRESENT AND FUTURE RESEARCH NEEDS Maternal COVID-19 and the fetus}

Irrespective of fetal infection, maternal infection can impact the developing fetal immune system, for example in human immunodeficiency virus or hepatitis C virus. ${ }^{135,136}$ Short-term impact of maternal covid-19 on the fetus has been described in a few small cohorts. In 51 maternal COVID-19 cases in the third trimester, no effects on neonatal cellular or humoral immunity or cytokine production were found, with the exception of IL- 6 and IL$10 .^{137}$ One neonate with extremely elevated IL-6, however, developed necrotizing enterocolitis. Pre-print data from a casecontrol study showed increased fetal inflammatory markers and cytokine functionality in neonates of mothers exposed to SARSCoV-2, without signs of neonatal infection. ${ }^{138}$ Overall current evidence is scarce and contradicting. It is important to understand if this impact on the fetal immune profile is protective or harmful, if the impact is transient or long-lasting and if the trimester and timing of exposure is affecting this impact. Moreover, advice on maternal immunization remains unclear as pregnant women were excluded from vaccine trials. Besides monitoring maternal COVID19 , there is a dire need for risk-benefits analyses for immunization regarding both mother and child. ${ }^{139}$

\section{Neonatal immune response}

Comparison of the neonatal immune response to SARS-CoV-2 versus responses to RSV or influenza virus and identifying differences between the adult and the neonatal cellular and humoral response to SARS-CoV-2 in larger cohorts could provide useful information on COVID-19 pathogenesis. This might become even more essential in light of future evolving virus strains with increasing virulence.

Another opportunity for future research is the potential of breastmilk. As outlined above, there appear to be associations with immunomodulator promotion via breastmilk, we still lack full understanding of the role of $\lg A$, secretory $\lg \mathrm{A}$ as a first-line defense in the mucosa, which may potentially be a more potent neutralizer of SARS-CoV-2 than serum IgG. ${ }^{140}$ With global rising antibody prevalence due to active infection or vaccination including in women of childbearing age, understanding protective components of breastmilk could provide a useful tool in future passive immunization or even therapeutic use.

\section{Long-term sequelae}

It is not known if maternal or mild neonatal COVID-19 potentially cause serious long-term sequelae and therefore follow-up of antenatally and neonatally exposed infants is necessary. As discussed previously, the impact of maternal COVID-19 on the fetus and its clinical significance needs to be addressed, as potential (neurodevelopmental) sequelae has not yet been ruled out. 
Although neonates mainly experience mild acute symptoms, across the globe, a subset of patients who sustain an acute SARSCoV-2 infection are developing a wide range of persistent symptoms, also in children. ${ }^{141,142}$ These complaints can last up to a few weeks, but sometimes several months and even 1 year after an infection with COVID-19. These patients are being given the diagnosis Long COVID or Post-acute sequelae of COVID-19 (PASC). In The Netherlands, a nationwide multicentre hospitalbased prospective cohort study, called the Clinical features of COVID-19 in Pediatric Patients (COPP)-study, was initiated early $2020 .^{143}$ This study is extended by the COPP2 study, which looks into the long-term sequelae of pediatric COVID-19. With respect to neonatal COVID-19, it is important to focus on neurodevelopmental aspects in light of the neurotropic potential of the SARSCoV-2 virus in other age groups.

It is very likely that SARS-CoV-2 will be a continuous global threat despite effective vaccination programs. Reasons for this include that it has become obvious that the virus can rapidly mutate and become more contagious, and the vaccination program in low- and middle-income countries continues to lag behind. It poses multiple threats to the health and well-being of children worldwide. ${ }^{14} \mathrm{~A}$ better understanding of age-group-specific disease pathomechanisms, treatment options including effect of infant/child vaccinations, long-term outcomes and prevention of viral spread is urgently needed and should be the topics of future research. ${ }^{144,145}$ The overall low morbidity of SARS-CoV-2 infection in neonates as compared to adults is striking, as well as the low susceptibility to SARS-CoV-2 compared to bacterial or other severe viral infections. The relatively low incidence among children, and especially neonates, necessitate multi-national collaborations ${ }^{128}$ to address these questions in adequately sized studies. International registries $^{146}$ and collaborations represent first vital steps in this direction, but more work is needed. The very successful RECOVERY trial, by far the largest double-blind controlled trial of hospitalized patients with COVID-19, included pregnant women, infants and children. This trial, which, with appropriate administrative prioritization and support of all regulatory agencies, went from inception to recruitment in only 9 days, could serve as an ideal model for future platform trials. ${ }^{147}$ To achieve this, current pathways of collaboration need further nurturing, for which adequate public support and funding remain mandatory necessities. Following this example, national and international healthcare policy makers need to remain prepared to provide structural and financial support for the research needs in child health during the current and for future pandemics.

Future research should focus on three main areas of interest: what is the impact of maternal COVID-19 on the fetus, what does the efficient neonatal immune response teach us on COVID-19 pathophysiology, and could maternal or mild neonatal COVID-19 potentially cause serious long-term sequelae?

\section{CONCLUSION}

In conclusion, while there is a considerable amount of evidence available related to the COVID-19 pandemic and neonatal care, there are still many unanswered questions. The evidence to date should be used to continue to promote best practice in neonatal care, with particular consideration to revising policies that have now been shown to negatively impact rather than protect our patients. It is important to look forward to developing effective research strategies to address ongoing concerns in relation to neonatal care in the COVID-19 pandemic.

\section{REFERENCES}

1. Kitano, T. et al. The differential impact of pediatric COVID-19 between highincome countries and low- and middle-income countries: A systematic review of fatality and ICU admission in children worldwide. PLOS ONE 16, e0246326 (2021).
2. Kirmani, S. \& Saleem, A. Impact of COVID-19 pandemic on paediatric services at a referral centre in Pakistan: lessons from a low-income and middle-income country setting. Arch. Dis. Child. https://doi.org/10.1136/archdischild-2020319424 (2020).

3. Duke, T., English, M., Carai, S. \& Qazi, S. Paediatric care in the time of COVID-19 in countries with under-resourced healthcare systems. Arch. Dis. Child 105, 616-617 (2020).

4. Klingenberg, C. et al. International Neonatal COVID-19 Consortium. COVID-19 preparedness-a survey among neonatal care providers in low- and middleincome countries. J. Perinatol. 41, 988-997 (2021).

5. Roberton, T. et al. Early estimates of the indirect effects of the COVID-19 pandemic on maternal and child mortality in low-income and middle-income countries: a modelling study. Lancet Glob. Health 8, e901-e908 (2020).

6. Cyranoski, D. Why children avoid the worst coronavirus complications might lie in their arteries. Nature 582, 324-325 (2020).

7. Vousden, N. et al. The incidence, characteristics and outcomes of pregnant women hospitalized with symptomatic and asymptomatic SARS-CoV-2 infection in the UK from March to September 2020: a national cohort study using the UK Obstetric Surveillance System (UKOSS). Preprint at medRxiv https://doi.org/ 10.1101/2021.01.04.21249195 (2021).

8. Götzinger, F., Santiago-Garcia, B., Fumadó-Pérez, V., Brinkmann, F. \& Tebruegge, M. ptbnet COVID-19 Study Group. The ability of the neonatal immune response to handle SARS-CoV-2 infection. Lancet Child Adolesc Health. 5, e6-e7 (2021).

9. Gale, C. et al. Characteristics and outcomes of neonatal SARS-CoV-2 infection in the UK: a prospective national cohort study using active surveillance. Lancet Child Adolesc. Health https://doi.org/10.1016/S2352-4642(20)30342-4 (2020).

10. Pareek, M. et al. Ethnicity and COVID-19: an urgent public health research priority. Lancet 395, 1421-1422 (2020).

11. Saheb Sharif-Askari, N. et al. Airways expression of SARS-CoV-2 receptor, ACE2, and TMPRSS2 is lower in children than adults and increases with smoking and COPD. Mol. Ther. Methods Clin. Dev. 18, 1-6 (2020).

12. Soraya, G. V. \& Ulhaq, Z. S. Interleukin-6 levels in children developing SARS-CoV2 infection. Pediatr. Neonatol. 61, 253-254 (2020).

13. Lindan, C. E. et al. Neuroimaging manifestations in children with SARS-CoV-2 infection: a multinational, multicentre collaborative study. Lancet Child Adolesc. Health 5, 167-177 (2021).

14. Klein, J. D. et al. Promoting and supporting children's health and healthcare during COVID-19-International Paediatric Association Position Statement. Arch. Dis. Child 105, 620-624 (2020).

15. Michelow, I. C., Oladokun, R. E., Torbunde, N. \& Sam-Agudu, N. A. Things must not fall apart: the ripple effects of the COVID-19 pandemic on children in subSaharan Africa. Pediatr. Res. https://doi.org/10.1038/s41390-020-01174-y (2020).

16. Semaan, A. et al. Voices from the frontline: findings from a thematic analysis of a rapid online global survey of maternal and newborn health professionals facing the COVID-19 pandemic. BMJ Glob. Health 5, e002967 (2020).

17. Dureab, F., Al-Awlaqi, S. \& Jahn, A. COVID-19 in Yemen: preparedness measures in a fragile state. Lancet Public Health 5, e311 (2020).

18. Ashish, K. C. et al. Effect of the COVID-19 pandemic response on intrapartum care, stillbirth, and neonatal mortality outcomes in Nepal: a prospective observational study. Lancet Glob. Health 8, e1273-e1281 (2020).

19. Horton, R. Offline: COVID-19 and the NHS- "a national scandal". Lancet $\mathbf{3 9 5}$, 1022 (2020).

20. Oppenheim, B. et al. Assessing global preparedness for the next pandemic: development and application of an Epidemic Preparedness Index. BMJ Glob. Health 4, e001157 (2019).

21. Remuzzi, A. \& Remuzzi, G. COVID-19 and Italy: what next? Lancet 395, 1225-1228 (2020).

22. Walker, P. G. T. et al. The impact of COVID-19 and strategies for mitigation and suppression in low- and middle-income countries. Science 369, 413-422 (2020).

23. Allotey, J. et al. Clinical manifestations, risk factors, and maternal and perinatal outcomes of coronavirus disease 2019 in pregnancy: living systematic review and meta-analysis. BMJ 370, m3320 (2020).

24. WHO Team (Sexual and Reproductive Health and Research, WHO Headquarters). Definition and categorisation of the timing of mother-to-child transmission of SARS-CoV-2. WHO Scientific Brief https://www.who.int/publications/i/item/ WHO-2019-nCoV-mother-to-child-transmission-2021.1 (2021).

25. Stowe, J. et al. Stillbirths during the COVID-19 pandemic in England, April-June 2020. JAMA 325, 86-87 (2021).

26. Delahoy, M. J. et al.Characteristics and maternal and birth outcomes of hospitalized pregnant women with laboratory-confirmed COVID-19 - COVID-NET, 13 States, March 1-August 22, 2020. MMWR Morb. Mortal. Wkly Rep. 69, 1347-1354 (2020).

27. Panagiotakopoulos, L. et al. SARS-CoV-2 infection among hospitalized pregnant women: reasons for admission and pregnancy characteristics-eight U.S. Health 
Care Centers, March 1-May 30, 2020. MMWR Morb. Mortal. Wkly Rep. 69, 1355-1359 (2020)

28. Vivanti, A. J. et al. Transplacental transmission of SARS-CoV-2 infection. Nat. Commun. 11, 3572 (2020).

29. Correia, C. R. et al. Congenital SARS-CoV-2 infection in a neonate with severe acute respiratory syndrome. Pediatr. Infect. Dis. J. 39, e439-e443 (2020).

30. Hopwood, A. J. et al. Severe acute respiratory syndrome coronavirus-2 pneumonia in a newborn treated with remdesivir and coronavirus disease 2019 convalescent plasma. J. Pediatr. Infect. Dis. Soc. 10, 691-694 (2021).

31. Alzamora, M. C. et al. Severe COVID-19 during pregnancy and possible vertical transmission. Am. J. Perinatol. 37, 861-865 (2020).

32. Patanè, L. et al. Vertical transmission of coronavirus disease 2019: severe acute respiratory syndrome coronavirus 2 RNA on the fetal side of the placenta in pregnancies with coronavirus disease 2019-positive mothers and neonates at birth. Am. J. Obstet. Gynecol. Mfm. 2, 100145 (2020).

33. Algarroba, G. N. et al. Visualization of severe acute respiratory syndrome coronavirus 2 invading the human placenta using electron microscopy. Am. J. Obstet. Gynecol. 223, 275-278 (2020).

34. Hosier, H. et al. SARS-CoV-2 infection of the placenta. J. Clin. Invest. 130, 4947-4953 (2020).

35. Schoenmakers, S. et al. Severe acute respiratory syndrome coronavirus 2 placental infection and inflammation leading to fetal distress and neonatal multiorgan failure in an asymptomatic woman. J. Pediatr. Infect. Dis. Soc. 10, 556-561 (2021).

36. Wong, Y. P., Khong, T. Y. \& Tan, G. C. The effects of COVID-19 on placenta and pregnancy: what do we know so far? Diagnostics 11, 94 (2021).

37. Gengler, C. et al. SARS-CoV-2 ACE-receptor detection in the placenta throughout pregnancy. Clin. Microbiol. Infect. 27, 489-490 (2021).

38. van Doorn, A. S., Meijer, B., Frampton, C. M. A., Barclay, M. L. \& de Boer, N. K. H. Systematic review with meta-analysis: SARS-CoV-2 stool testing and the potential for faecal-oral transmission. Aliment Pharm. Ther. 52, 1276-1288 (2020).

39. Cimolai, N. A comprehensive analysis of maternal and newborn disease and related control for COVID-19. SN Compr. Clin. Med. 17, 1-23 (2021).

40. Cavicchiolo, M. E. et al. Universal screening of high-risk neonates, parents, and staff at a neonatal intensive care unit during the SARS-CoV-2 pandemic. Eur. J. Pediatr. 179, 1949-1955 (2020).

41. Sutton, H. J., Dargaville, P. A. \& Spotswood, N. E. Unravelling the epidemiology and clinical impact of SARS-CoV-2 infection in neonates. Acta Paediatr. 110, 2482-2483 (2021)

42. Kharrat, A., Neish, A., Diambomba, Y. \& Jain, A. Non-COVID co-morbidity: potential indirect consequences of the SARS-CoV-2 pandemic in a neonatal intensive care unit. J. Hosp. Infect. 109, 65-67 (2021).

43. More, K. et al. Outcomes of neonates born to mothers with coronavirus disease 2019 (COVID-19)—National Neonatology Forum (NNF) India COVID-19 Registry. Indian Pediatr. 58, 525-531 (2021).

44. Ciapponi, A. et al. COVID-19 and pregnancy: an umbrella review of clinical presentation, vertical transmission, and maternal and perinatal outcomes. PLoS ONE 16, e0253974 (2021).

45. Raschetti, R. et al. Synthesis and systematic review of reported neonatal SARSCoV-2 infections. Nat. Commun. 11, 5164 (2020).

46. Conti, M. G. et al. Consequences of early separation of maternal-newborn dyad in neonates born to SARS-CoV-2 positive mothers: an observational study. Int. J. Environ. Res. Public Health 18, 5899 (2021).

47. Griffin, l. et al. The impact of COVID-19 infection on labor and delivery, newborn nursery, and neonatal intensive care unit: prospective observational data from a single hospital system. Am. J. Perinatol. 37, 1022-1030 (2020).

48. Mayopoulos, G. A., Ein-Dor, T., Li, K. G., Chan, S. J. \& Dekel, S. COVID-19 positivity associated with traumatic stress response to childbirth and no visitors and infant separation in the hospital. Sci. Rep. 11, 13535 (2021).

49. Sakalidis, V. S. et al. Wellbeing of breastfeeding women in Australia and New Zealand during the COVID-19 pandemic: a cross-sectional study. Nutrients 13, 1831 (2021).

50. Ronchi, A. et al. Evaluation of rooming-in practice for neonates born to mothers with severe acute respiratory syndrome coronavirus 2 infection in Italy. JAMA Pediatr. 175, 260-266 (2021).

51. Kaufman, D. A. \& Puopolo, K. M. Infants born to mothers with COVID-19-making room for rooming-in. JAMA Pediatr. 175, 240-242 (2021).

52. Song, D. et al. Passive and active immunity in infants born to mothers with SARS-CoV-2 infection during pregnancy: prospective cohort study. BMJ Open 11, e053036 (2021)

53. Egerup, P. et al. Severe acute respiratory syndrome coronavirus 2 (SARS-CoV-2) antibodies at delivery in women, partners, and newborns. Obstet. Gynecol. 137, 49-55 (2021).

54. Flannery, D. D. et al. Assessment of maternal and neonatal cord blood SARS-CoV-2 antibodies and placental transfer ratios. JAMA Pediatr. 175, 594-600 (2021).
55. Edlow, A. G. et al. Assessment of maternal and neonatal SARS-CoV-2 viral load, transplacental antibody transfer, and placental pathology in pregnancies during the COVID-19 pandemic. JAMA Netw. Open 3, e2030455 (2020).

56. Munoz, F. M. Can we protect pregnant women and young infants from COVID19 through maternal immunization? JAMA Pediatr. 175, 561-562 (2021).

57. Gill, L. \& Jones, C. W. Severe acute respiratory syndrome coronavirus 2 (SARSCoV-2) antibodies in neonatal cord blood after vaccination in pregnancy. Obstet. Gynecol. https://doi.org/10.1097/AOG.0000000000004367 (2021).

58. Zdanowski, W. \& Waśniewski, T. Evaluation of SARS-CoV-2 spike protein antibody titers in cord blood after COVID-19 vaccination during pregnancy in Polish healthcare workers: preliminary results. Vaccines 9, 675 (2021).

59. Groß et al. Detection of SARS-CoV-2 in human breastmilk. Lancet 395 , 1757-1758 (2020). Erratum in: Lancet 396, 758 (2020).

60. Kumar, J., Meena, J., Yadav, A. \& Kumar, P. SARS-CoV-2 detection in human milk: a systematic review. J. Matern. Fetal Neonatal Med. 1-8 https://doi.org/10.1080/ 14767058.2021.1882984 (2021). Epub ahead of print.

61. Kilic, T., Kilic, S., Berber, N. K., Gunduz, A. \& Ersoy, Y. Investigation of SARS-CoV-2 RNA in milk produced by women with COVID-19 and follow-up of their infants: a preliminary study. Int. J. Clin. Pr. 75, e14175 (2021).

62. Lugli, L. et al. An uninfected preterm newborn inadvertently fed SARS-CoV-2positive breast milk. Pediatrics 146, e2020004960 (2020).

63. Prasad, A. et al. Excretion of SARS-CoV-2 in breast milk: a single-centre observational study. BMJ Paediatr. Open 5, e001087 (2021).

64. Centeno-Tablante, E. et al. Transmission of SARS-CoV-2 through breast milk and breastfeeding: a living systematic review. Ann. N. Y. Acad. Sci. 1484, 32-54 (2021).

65. Kunjumon, B. et al. Breast milk and breastfeeding of infants born to SARS-CoV-2 positive mothers: a prospective observational cohort study. Am. J. Perinatol. https://doi.org/10.1055/s-0041-1731451 (2021).

66. Shlomai, N. O. et al. Neonatal SARS-CoV-2 infections in breastfeeding mothers. Pediatrics 147, e2020010918 (2021).

67. Fox, A. et al. Robust and specific secretory IgA against SARS-CoV-2 detected in human milk. iScience 23, 101735 (2020).

68. Demers-Mathieu, V., DaPra, C., Fels, S. \& Medo, E. Receptor-binding domain severe acute respiratory syndrome coronavirus 2-specific antibodies in human milk from mothers with coronavirus disease 2019 polymerase chain reaction or with symptoms suggestive of coronavirus disease 2019. J. Pediatr. Gastroenterol. Nutr. 73, 125-128 (2021).

69. Demers-Mathieu, V. et al. Human milk antibodies against S1 and S2 subunits from SARS-CoV-2, HCoV-OC43, and HCoV-229E in mothers with a confirmed COVID-19 PCR, viral SYMPTOMS, and unexposed mothers. Int. J. Mol. Sci. 22, 1749 (2021).

70. Demers-Mathieu, V., DaPra, C. \& Medo, E. Comparison of severe acute respiratory syndrome coronavirus 2-specific antibodies' binding capacity between human milk and serum from coronavirus disease 2019-recovered women. Breastfeed. Med. 16, 393-401 (2021).

71. Favara, D. M. et al. Detection of breastmilk antibodies targeting SARS-CoV-2 nucleocapsid, spike and receptor-binding-domain antigens. Emerg. Microbes Infect. 9, 2728-2731 (2020).

72. van Keulen, B. J. et al. Human milk from previously COVID-19-infected mothers: the effect of pasteurization on specific antibodies and neutralization capacity. Nutrients 13, 1645 (2021).

73. Low, J. M. et al. Titres and neutralising capacity of SARS-CoV-2-specific antibodies in human milk: a systematic review. Arch. Dis. Child Fetal Neonatal Ed. https://doi.org/10.1136/archdischild-2021-322156 (2021).

74. Fox, A., Norris, C., Amanat, F., Zolla-Pazner, S. \& Powell, R. L. The vaccine-elicited immunoglobulin profile in milk after COVID-19 mRNA-based vaccination is lgGdominant and lacks secretory antibodies. medRxiv $21253831 \mathrm{https}: / /$ doi.org/ 10.1101/2021.03.22.21253831 (2021).

75. Bobik, T. V. et al. Epitope-specific response of human milk immunoglobulins in COVID-19 recovered women. Pathogens 10, 705 (2021).

76. Pace, R. M. et al. Characterization of SARS-CoV-2 RNA, antibodies, and neutralizing capacity in milk produced by women with COVID-19. mBio 12, e0319220 (2021).

77. Britton, G. J. et al. Limited intestinal inflammation despite diarrhea, fecal viral RNA and SARS-CoV-2-specific IgA in patients with acute COVID-19. Sci. Rep. 11, 13308 (2021).

78. Malshe, N. et al. Perinatal transmission of SARS-CoV-2 and transfer of maternal $\mathrm{IgG} /$ neutralizing anti-SARS-CoV-2 antibodies from mothers with asymptomatic infection during pregnancy. Infection https://doi.org/10.1007/s15010-02101650-5. (2021).

79. Lang, J. et al. Inhibition of SARS pseudovirus cell entry by lactoferrin binding to heparan sulfate proteoglycans. PLOS ONE 6, e23710 (2011).

80. Peroni, D. G. \& Fanos, V. Lactoferrin is an important factor when breastfeeding and COVID-19 are considered. Acta Paediatr. 109, 2139-2140 (2020). 
81. Vassilopoulou, E. et al. Breastfeeding and COVID-19: from nutrition to immunity. Front. Immunol. 12, 661806 (2021).

82. Bardanzellu, F., Puddu, M. \& Fanos, V. Breast milk and COVID-19: from conventional data to "omics" technologies to investigate changes occurring in SARS-CoV-2 positive mothers. Int. J. Environ. Res. Public Health 18, 5668 (2021).

83. Briana, D. D. et al. Early human milk lactoferrin during SARS-CoV-2 infection. J. Matern. Fetal Neonatal Med. https://doi.org/10.1080/14767058.2021.1920010 (2021).

84. Unger, S. et al. Holder pasteurization of donated human milk is effective in inactivating SARS-CoV-2. CMAJ 192, E871-E874 (2020).

85. Mitoulas, L. R., Schärer-Hernández, N. G. \& Liabat, S. Breastfeeding, human milk and COVID-19-what does the evidence say? Front. Pediatr. 8, 613339 (2020).

86. Collier, A. Y. et al. Immunogenicity of COVID-19 mRNA vaccines in pregnant and lactating women. JAMA 325, 2370-2380 (2021).

87. Gray, K. J. et al. Coronavirus disease 2019 vaccine response in pregnant and lactating women: a cohort study. Am. J. Obstet. Gynecol. 225, 303.e1-303.e17 (2021).

88. Friedman, M. R. et al. BNT162b2 COVID-19 mRNA vaccine elicits a rapid and synchronized antibody response in blood and milk of breastfeeding women. Preprint at medRxiv 21252603 https://doi.org/10.1101/2021.03.06.21252603 (2021).

89. Esteve-Palau, E. et al. Quantification of specific antibodies against SARS-CoV-2 in breast milk of lactating women vaccinated with an mRNA vaccine. Preprint at medRxiv 21254819 https://doi.org/10.1101/2021.04.05.21254819 (2021).

90. Juncker, H. G. et al. The levels of SARS-CoV-2 specific antibodies in human milk following vaccination. J. Hum. Lact. 37, 477-484 (2021).

91. Golan, Y. et al. Evaluation of Messenger RNA From COVID-19 BTN162b2 and mRNA-1273 Vaccines in Human Milk. JAMA Pediatr. 175, 1069-1071 (2021).

92. Bertrand, K., Honerkamp-Smith, G. \& Chambers, C. Maternal and child outcomes reported by breastfeeding women following mRNA COVID-19 vaccination. Preprint at medRxiv https://doi.org/10.1101/2021.04.21.21255841 (2021).

93. McLaurin-Jiang, S., Garner, C. D., Krutsch, K. \& Hale, T. W. Maternal and child symptoms following COVID-19 vaccination among breastfeeding mothers. Breastfeed. Med. https://doi.org/10.1089/bfm.2021.0079 (2021).

94. Sandberg, J. T. et al. SARS-CoV-2-specific humoral and cellular immunity persists through 9 months irrespective of COVID-19 severity at hospitalisation. Clin. Transl. Immunol. 10, e1306 (2021).

95. van Veenendaal, N. R., Deierl, A., Bacchini, F., O'Brien, K. \& Franck L. S. International Steering Committee for Family Integrated Care. Supporting parents as essential care partners in neonatal units during the SARS-CoV-2 pandemic. Acta Paediatr. 110, 2008-2022 (2021).

96. Zhang, Y., Sun, Z., Latour, J. M., Hu, B. \& Qian, J. Hospital response to the COVID19 outbreak: the experience in Shanghai, China. J. Adv. Nurs. 76, 1483-1485 (2020).

97. Scala, M., Marchman, V. A., Brignoni-Pérez, E., Morales, M. C. \& Travis, K. E. Impact of the COVID-19 pandemic on developmental care practices for infants born preterm. Pediatrics https://doi.org/10.1101/2020.11.25.20238956 (2020).

98. Darcey-Mahoney, A. et al. Impact of restrictions on parental presence in neonatal intensive care units related to COVID-19. Pediatrics https://doi.org/10.1101/ 2020.07.22.20158949 (2020)

99. Salvatore, C. M. et al. Neonatal management and outcomes during the COVID19 pandemic: an observation cohort study. Lancet Child Adolesc. Health 4, 721-727 (2020).

100. Brown, A. \& Shenker, N. Experiences of breastfeeding during COVID-19: lessons for future practical and emotional support. Matern. Child Nutr. https://doi.org/ 10.1111/mcn.13088 (2021).

101. Ding, X. et al. Effects of family-centered care interventions on preterm infants and parents in neonatal intensive care units: a systematic review and metaanalysis of randomized controlled trials. Aust. Crit. Care 32, 63-75 (2019).

102. Muniraman, H. et al. Parental perceptions of the impact of neonatal unit visitation policies during COVID-19 pandemic. BMJ Paediatric Open 4, e000899 (2020).

103. Bembich, S. et al. Parents experiencing NICU visit restrictions due to COVID-19 pandemic. Acta Paediatr. 110, 940-941 (2021).

104. Minckas, N. et al. Preterm care during the COVID-19 pandemic: A comparative risk analysis of neonatal deaths averted by kangaroo mother care versus mortality due to SARS-CoV-2 infection. EClinicalMedicine 33, 100733 (2021).

105. European Foundation for the Care of Newborn Infants. GLANCE releases new video series for Zero Separation. https://www.efcni.org/news/glance-releasesnew-video-series-for-zero-separation/ (2020).

106. Zhu, L. H. et al. Family-centered care improves clinical outcomes of very-lowbirth-weight infants: a quasi-experimental study. Front. Pediatrics 7, 138 (2019).

107. Oude Maatman, S. M. et al. Factors influencing implementation of familycentered care in a neonatal intensive care unit. Front. Pediatr. 8, 222 (2020).
108. WHO. Causes of newborn mortality and morbidity in the European Region. https://www.euro.who.int/en/health-topics/Life-stages/maternal-and-newbornhealth/causes-of-newborn-mortality-and-morbidity-in-the-european-region (2020).

109. Olin, A. et al. Stereotypic immune system development in newborn children. Cell 174, 1277-1292.e14 (2018).

110. Dowling, D. J. \& Levy, O. Ontogeny of early life immunity. Trends Immunol. 35, 299-310 (2014).

111. Yuanyuan, D. et al. Epidemiology of COVID-19 among children in China. Pediatrics 145, e20200702 (2020).

112. Pou, C. et al. The repertoire of maternal anti-viral antibodies in human newborns. Nat. Med. 25, 591-596 (2019).

113. Fonseca, W., Lukacs, N. W. \& Ptaschinski, C. Factors affecting the immunity to respiratory syncytial virus: from epigenetics to microbiome. Front. Immunol. 9, 226 (2018).

114. Yoon, H. E. et al. Age-associated changes in the vascular renin-angiotensin system in mice. Oxid. Med. Cell Longev. 2016, 6731093 (2016).

115. South, A. M., Tomlinson, L., Edmonston, D., Hiremath, S. \& Sparks, M. A. Controversies of renin-angiotensin system inhibition during the COVID-19 pandemic. Nat. Rev. Nephrol. https://doi.org/10.1038/s41581-020-0279-4 (2020).

116. O'Hare, F. M., William Watson, R. \& Molloy, E. J. Toll-like receptors in neonatal sepsis. Acta Paediatr. 102, 572-578 (2013).

117. Levy, O. et al. The adenosine system selectively inhibits TLR-mediated TNF-alpha production in the human newborn. J. Immunol. 177: 1956-1966 (2006).

118. Chantry, C. J., Howard, C. R. \& Auinger, P. Full breastfeeding duration and associated decrease in respiratory tract infection in US children. Pediatrics 117 , 425-432 (2006).

119. Fox, A. et al. Evidence of a significant secretory-IgA-dominant SARS-CoV-2 immune response in human milk following recovery from COVID-19. medRxiv 20089995 https://doi.org/10.1101/2020.05.04.20089995 (2020).

120. Kollmann, T. R., Kampmann, B., Mazmanian, S. K., Marchant, A. \& Levy, O. Protecting the newborn and young infant from infectious diseases: lessons from immune ontogeny. Immunity 46, 350-363 (2017).

121. Zimmermann, P. \& Curtis, N. Why is COVID-19 less severe in children? A review of the proposed mechanisms underlying the age-related difference in severity of SARS-CoV-2 infections. Arch. Dis. Child. https://doi.org/10.1136/archdischild2020-320338 (2020).

122. Walker, K. F., O'Donoghue, K. \& Grace, N. Maternal transmission of SARS-COV-2 to the neonate, and possible routes for such transmission: a systematic review and critical analysis. BJOG 127, 1324-1336 (2020).

123. Carsetti, R. et al. The immune system of children: the key to understanding SARS-CoV-2 susceptibility? Lancet Child Adolesc. Health 4, 414-416 (2020).

124. Goenka, A. et al. Young infants exhibit robust functional antibody responses and restrained IFN- $\gamma$ production to SARS-CoV-2. Cell Rep. Med. 2, 100327 (2021).

125. Godfred-Cato, S. et al. COVID-19-associated multisystem inflammatory syndrome in children-United States, March-July 2020. MMWR Morb. Mortal. Wkly Rep. 69, 1074-1080 (2020).

126. Sankaran, D., Nakra, N., Cheema, R., Blumberg, D. \& Lakshminrusimha, S. Perinatal SARS-CoV-2 infection and neonatal COVID-19: a 2021 update. Neoreviews 22, e284-e295 (2021).

127. Huntley, B. J. F. et al. Rates of maternal and perinatal mortality and vertical transmission in pregnancies complicated by severe acute respiratory syndrome coronavirus 2 (SARS-Co-V-2) infection: a systematic review. Obstet. Gynecol. 136, 303-312 (2020).

128. Molloy, E. J. et al. Neonates in the COVID-19 pandemic. Pediatr. Res. https://doi. org/10.1038/s41390-020-1096-y (2020).

129. Lavizzari, A. et al. International comparison of quidelines for managing neonates at the early phase of the SARS-CoV-2 pandemic. Pediatr. Res. https://doi.org/ 10.1038/s41390-020-0976-5 (2020).

130. Molloy, E. J. The doctor's dilemma: lessons from GB Shaw in a modern pandemic COVID-19. Pediatr. Res. https://doi.org/10.1038/s41390-020-0927-1 (2020).

131. Molloy, E. J. \& Murphy, N. Vitamin D, Covid-19 and children. Ir. Med. J. 113, 64 (2020).

132. Molloy, E. J. \& Bearer, C. F. COVID-19 in children and altered inflammatory responses. Pediatr. Res. 88, 340-341 (2020).

133. Pawar, R. et al. Neonatal multisystem inflammatory syndrome (MIS-N) associated with prenatal maternal SARS-CoV-2: a case series. Children 8, 572 (2021).

134. Horby, P. et al. Dexamethasone in hospitalized patients with Covid-19. N. Engl. J. Med. 384, 693-704 (2021).

135. Abu-Raya, B., Kollmann, T. R., Marchant, A. \& MacGillivray, D. M. The immune system of HIV517 exposed uninfected infants. Front. Immunol. 7, 383 (2016).

136. Babik, J. M., Cohan, D., Monto, A., Hartigan-O'Connor, D. J. \& McCune, J. M. The human fetal 519 immune response to hepatitis $C$ virus exposure in utero. $J$. Infect. Dis. 203, 196-206 (2011). 
137. Liu, P. et al. The immunologic status of newborns born to SARS-CoV-2-infected mothers in Wuhan, China. J. Allergy Clin. Immunol. 146, 101-109.e1 (2020).

138. Gee, S. et al. The legacy of maternal SARS-CoV-2 infection on the immunology of the neonate. Nat. Immunol. 22, 1490-1502 (2021).

139. Bardají, A. et al. The need for a global COVID-19 maternal immunisation research plan. Lancet 397, e17-e18 (2021).

140. Wang, Z. et al. Enhanced SARS-CoV-2 neutralization by dimeric IgA. Sci. Transl. Med. 13, eabf1555 (2021).

141. Peghin, M. et al. Post-COVID-19 symptoms 6 months after acute infection among hospitalized and non-hospitalized patients. Clin. Microbiol. Infect. 27, 1507-1513 (2021).

142. Brackel, C. L. H. et al. Pediatric long-COVID: an overlooked phenomenon? Pediatr. Pulmonol. https://doi.org/10.1002/ppul.25521 (2021).

143. Introduction to COPP Study. COPP Study 2021 https://www.covidkids.nl/englishintro/ (2021).

144. Fleming, P. F. et al. Paediatric research in the times of COVID-19. Pediatr. Res. https://doi.org/10.1038/s41390-021-01479-6 (2021).

145. Malhotra, A., Kumar, A., Roehr, C. C. \& den Boer, M. C. Inclusion of children and pregnant women in COVID-19 intervention trials. Pediatr. Res. 89, 1063-1064 (2021).

146. de Winter, J. P., De Luca, D. \& Tingay, D. G. COVID-19 surveillance for all newborns at the NICU; conditio sine qua non? Eur. J. Pediatr. 179, 1945-1947 (2020).

147. Fraser, A. G., Szymański, P., Macintyre, E. \& Landray, M. Regulating drugs, medical devices, and diagnostic tests in the European Union: early lessons from the COVID-19 pandemic? Eur. Heart J. 41, 2140-2144 (2020).

\section{AUTHOR CONTRIBUTIONS}

All listed authors substantially contributed to the content of the article, contributing individual sections and critical appraisal and editing.

\section{FUNDING INFORMATION}

This series is funded by ESPR.

\section{COMPETING INTERESTS}

The authors declare no competing interests.

\section{ADDITIONAL INFORMATION}

Correspondence and requests for materials should be addressed to Eleanor J. Molloy.

Reprints and permission information is available at http://www.nature.com/reprints

Publisher's note Springer Nature remains neutral with regard to jurisdictional claims in published maps and institutional affiliations. 